\title{
Editorials
}

\section{Improving the care of adolescents in general practice}

\section{INTRODUCTION}

There is a puzzling gap between the high prevalence of mental health disorders in adolescents and the low detection rates by GPs. ${ }^{1}$ From the point of view of a physician in child and adolescence psychiatry, it seems necessary to explore this area to prevent, identify, and treat health problems in this patient population as early and appropriately as possible. Even though adolescents may be vulnerable due to a great amount of biological, psychological, and social transformations in their lives, they also have the potential for significant and long-lasting effects of healthcare interventions. Therefore, supportive and well-organised interventions in this period of life may reduce the burden of mental disorders in adult life. The articles in this issue highlight the important role that general practice plays in this clinical area.

\section{THE BURDEN OF MENTAL HEALTH DISORDERS IN PRIMARY CARE}

Overall, the prevalence of mental health and psychosocial problems in primary care is high. ${ }^{2}$ Despite this, there is a scarcity of evidence on which treatments to prefer, and the generalisability of findings to other settings or countries has also to be clarified.

The need for early identification of mental health problems has been highlighted by a recent study indicating that subthreshold depression is a precursor to major depressive disorder in children and adolescents. ${ }^{3}$ Even though patients with subthreshold depression do not fulfil all diagnostic criteria of major depressive disorder, the impact of subthreshold depression appears to be comparable with the poor outcomes of major depressive disorder regarding psychiatric morbidity, functional impairment, and health service use. Furthermore, childhood-onset major depressive disorder displays longer depressive episodes, higher recurrence, more frequent hospitalisation, and more suicidality compared to adult-onset major depressive disorder. ${ }^{4}$ When almost $25 \%$ of adolescents attending primary care have been found to suffer from subthreshold depression, ${ }^{3}$ these findings together imply that GPs have a very challenging task of identifying and treating the right patients. At the same time, there is a general delay in seeking treatment for early onset

\section{"Childhood-onset major depressive disorder displays longer depressive episodes, higher recurrence, more frequent hospitalisation, and more suicidality compared to adult-onset major depressive disorder.}

mental disorders, as well as a lower overall probability of seeking help. ${ }^{5}$

\section{WHY DO ADOLESCENTS HESITATE TO SEEK HELP?}

Adolescents may be reluctant to seek care from the GP for several reasons. These reasons include embarrassment, concerns regarding confidentiality and consent, reticence about emotional distress, and former poor experiences of care. ${ }^{6}$ Knowledge of these potential barriers and of how to respond to them in the best possible way is one of the prerequisites for a youth-friendly general practice. The article by Aarseth et al ${ }^{6}$ examines the introduction of a low-cost, feasible approach for increasing GP-seeking behaviour by sending personal information letters to 16-year-olds. The letters addressed health issues of importance to adolescents to facilitate low threshold and enhanced accessibility of primary healthcare services. This intervention was easily administrated and significantly increased the proportion of youths in contact with a GP from 59\% to $69 \%$.

\section{THE ROLE OF EARLY IDENTIFICATION AND INTERVENTION STRATEGIES}

Several potential risk factors for subthreshold depression and major depressive disorder have been identified, including poor general health, stressful life events, and a high level of maternal depressive symptoms.? The known risk factors stress the need for selective preventive interventions targeting children, for example, those who suffer from chronic physical illness or children whose mothers present depressive symptoms. Contrary to these findings, most children with a psychiatric disorder from a high-risk sample of children of parents with depression were not in contact with services. ${ }^{8}$ Improving ease of access to services, increasing parental and professional awareness of mental health problems in children and adolescents, and improving links between different healthcare services may help early detection and intervention strategies. However, more future research is needed on how to apply screening instruments in general practice and subsequently treat symptoms of psychiatric disorders in children and adolescents.

\section{NEW MODELS OF MENTAL HEALTHCARE ACROSS THE PRIMARY-SECONDARY CARE INTERFACE}

Other articles in this issue explore the GP factors associated with engagement between GPs and adolescents. ${ }^{1.9}$ Findings from these studies show that uncertainty, GPs' views of adolescents and their health needs, and the knowledge frameworks employed by GPs when meeting patients affect the role of GPs in managing adolescents' psychological difficulties. New models of collaborative care and continuing

\section{"Collaborative care and new structures for working together across the borders of primary and secondary care may reveal new opportunities for responding adequately to the needs of adolescents.}


professional development are suggested to improve care of adolescents.

Previous studies have indicated that collaborative care is significantly able to influence the behaviours of health professionals in primary care and enhance patient outcomes such as psychiatric admissions and quality of life. 10,11 Likewise, active participation in postgraduate GP training has been associated with more positive patient experiences of care. ${ }^{12}$

Collaborative care and new structures for working together across the borders of primary and secondary care may reveal new opportunities for responding adequately to the needs of adolescents. Cross-sectoral cooperation could be a facilitator for continuing professional development and hence improvement of the services delivered by all involved health professionals: GPs, community workers, and employees of hospital care. However, more large, well designed, conducted and reported trials are required to answer the traditional 'What works for whom?' question and before any clinical or policymaking decisions concerning adolescent care can be made.

Future health services research should also include patient reported outcome measures in order to elucidate the patient perspective when evaluating clinical care. Apart from the more restricted assessment of the medical outcomes, effective improvement of care also entails assessment of the effect of services on the patient as a whole. ${ }^{13}$ For mental health disorders this is particularly important, since this kind of disease and treatment affect many aspects of a patients life. An example of the knowledge needed to deliver patient-centred care is provided by the aforementioned article by Aarseth where the authors mention adolescents' preferences regarding communication methods when engaging in healthcare. ${ }^{6}$

Collaborative care usually implies an increased number of health professionals involved in the care and treatment of each patient. ${ }^{13}$ This could also mean more transitions between healthcare providers or sites of care. Each transition point between providers or sites of care poses a risk of information being lost and subsequent problems, delays, or even flaws in care arising. This risk increases with the number of transition points in the care trajectory. Problems of fragmentation of services may also arise when patients have parallel care trajectories at different healthcare providers without providers sharing information or planning care.

\section{ADDRESS FOR CORRESPONDENCE}

\section{Louise Hyldborg Lundstrøm}

Centre for Child and Adolescent Psychiatry, The

Capital Region. Department. of Hillerød, the ADHD

clinic, Dyrehavevej 48, DK-3400, Hillerød

Denmark.

\section{E-mail: louiselundstroemagmail.com}

Furthermore, health professionals come from different organisational and educational backgrounds, which may compromise coordination of care and give rise to negative effects on outcomes. Therefore, when planning collaborative care, continuity of care must be kept in mind for delivering patient-centred care.

\section{CONCLUSIONS}

All in all, health care faces many challenges in adolescent mental health care. Health professionals and leaders have to wisely reshape service delivery aimed at this population, if we want to remove the barriers young people face in accessing care. However, let's get started solving these challenges for the benefit of our adolescents: they need it.

\section{Louise Hyldborg Lundstrøm.}

Centre for Child and Adolescent Psychiatry, The Capital Region, Department of Hillerød, the ADHD clinic, Hillerød, Denmark.

\section{Provenance}

Commissioned; not externally peer reviewed.

\section{DOI: 10.3399/bjgp14X679552}

\section{REFERENCES}

1. Roberts JH, Crosland A, Fulton J. Patterns of engagement between GPs and adolescents presenting with psychological difficulties: a qualitative study. Br J Gen Pract 2014; DOI: 10.3399/bjgp14X679660.

2. Bower P, Knowles S, Coventry PA, Rowland N. Counselling for mental health and psychosocial problems in primary care. Cochrane Database Syst Rev 2011; (9): CD001025.

3. Wesselhoeft R, Sorensen MJ, Heiervang ER, Bilenberg N. Subthreshold depression in children and adolescents - a systematic review. J Affect Disord 2013; 151(1): 7-22.

4. Korczak DJ, Goldstein BI. Childhood onset major depressive disorder: course of illness and psychiatric comorbidity in a community sample. J Pediatr 2009; 155(1): 118-123.

5. Wang PS, Berglund P, Olfson M, et al. Failure and delay in initial treatment contact after first onset of mental disorders in the National Comorbidity Survey Replication. Arch Gen Psychiatry 2005; 62(6): 603-613.

6. Aarseth S, Dalen I, Haavet OR. An effective approach to encouraging adolescents to contact their general practitioner: A community-based trial. Br J Gen Pract 2014; DOI: 10.3399/bjgp14X679688

7. Wesselhoeft R. Childhood depressive disorders [Thesis]. Mental Health Services in the Region of Southern Denmark and University of Southern Denmark; 2014.

8. Potter R, Mars B, Eyre O, et al. Missed opportunities: mental disorder in children of parents with depression. Br J Gen Pract 2012; DOI: 10.3399/bjgp12X652355.

9. Roberts JH, Crosland A, Fulton J. GPs' responses to adolescents presenting with psychological difficulties: a conceptual model of fixers, future planners, and collaborators. Br J Gen Pract 2014; DOI:10.3399/ bjgp14X679679.

10. Harkness EF, Bower PJ. On-site mental health workers delivering psychological therapy and psychosocial interventions to patients in primary care: effects on the professional practice of primary care providers. Cochrane Database Syst Rev 2009; (1): $\mathrm{CD} 000532$

11. Reilly S, Planner C, Gask L, et al. Collaborative care approaches for people with severe mental illness. Cochrane Database Syst Rev 2013; (11): CD009531.

12. Ashworth M, Schofield P, Durbaba S, Ahluwalia S. Patient experience and the role of postgraduate GP training: a crosssectional analysis of national Patient Survey data in England. Br J Gen Pract 2014; DOI: 10.3399/bjgp14X677545.

13. Lundstrøm LH. The patient perspective on continuity of cancer care [Thesis]. Faculty of health and medical sciences, University of Copenhagen, 2012. 